Journal of

Entreprenuership and

Project Management

(JEPM)

MONTE-CARLO APPROACH FOR MEASURING ADJUSTING COST RISKS VALUES OF RESIDENTIAL BUILDING PROJECT'S WHOLE LIFE CYCLE FROM CLIENTS' PERSPECTIVE IN THE UNITED ARAB EMIRATES

Journal of Entrepreneurship and Project Management, Vol. 6 No. 1 (2021), 72-89.

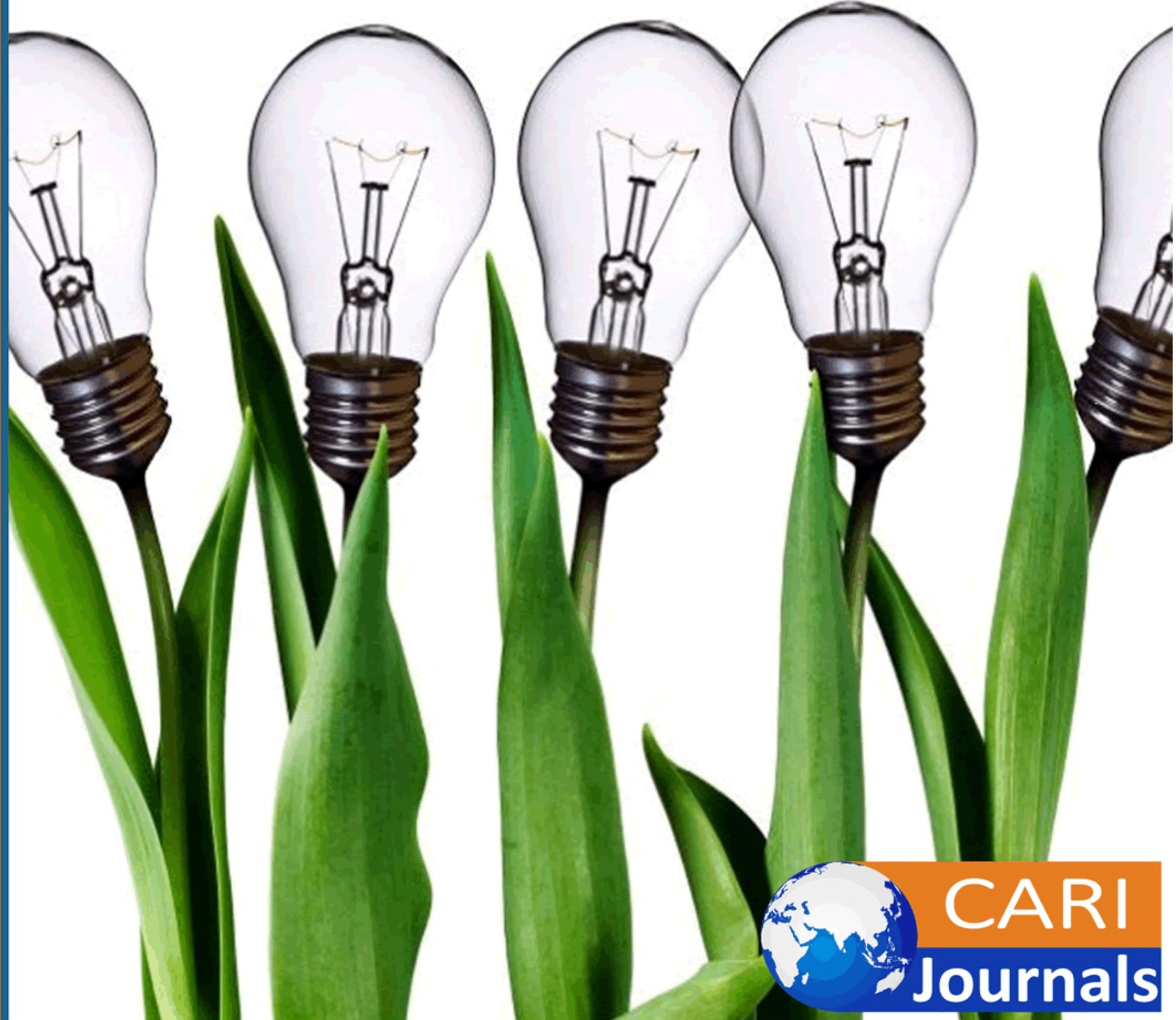




\title{
MONTE-CARLO APPROACH FOR MEASURING ADJUSTING COST RISKS VALUES OF RESIDENTIAL BUILDING PROJECT'S WHOLE LIFE CYCLE FROM CLIENTS' PERSPECTIVE IN THE UNITED ARAB EMIRATES
}

\author{
Ahmed Sadek \\ The British University in Dubai \\ 2015132021@student.buid.ac.ae
}

\begin{abstract}
Purpose - Although projects' experts always take into consideration the related cost-risks. They are experiencing the challenge of not being able to finish the project within the estimated budget. Latest cost-risks studies concentrated on modelling and estimating risks at the preconstruction stage. This article aims to approach Monte-Carlo simulation using stochastic mathematical modelling to measure cost-risks error (i.e., adjusting cost-risks).

Methodology - The approach of this research is solely quantitative. It is using statistical modelling and simulations to ensure the accuracy and precision of the developed Monte-Carlo model. However, this study is utilizing Microsoft Office Excel Software Mersenne twister algorithm to generate random numbers to ensure most accurate Monte-Carlo approach. The mathematical equations system is built into Excel.

Findings - The research outputs are considered significant in project management body of knowledge. This is because of the resulted evidence that is proving the applicability to measure cost risks error using Monte-Carlo simulation. This study presented cost risks and differentiated between contractors' and clients' views.

Unique contribution to theory, practice and policy - The originality of this article comes from providing the first Monte-Carlo approach for measuring projects' cost-risks error from client's perspective. The theoretical-implications, practical-implications, and limitations are presented in the conclusion for future research.
\end{abstract}

Keywords: Adjusting Cost-Risks, Monte-Carlo, Probability, Normal Distribution, Residential Buildings, Project's WLC, Mathematical Modeling, Relativity Change.

\section{INTRODUCTION}

In this article, the process of computing accurate risks' errors will be detailed. This research will conduct Monte-Carlo Simulation to compute the adjusted and adjusting risks used in the cost estimation modelling. Therefore, this article Monte-Carlo simulation will be based on taking 10,000 random measures for each risk factor (i.e., 117 final validated risks) to provide accurate cost estimation results.

It is important to know about the Monte Carlo approach's history to understand its value and importance (Jackel, 2002). In the 1940s, the Monte Carlo method for mathematical computation started by three American mathematicians (i.e., Nicholas Metropolis, John von Neumann, and Stanislav Ulam) during the entrance programable computers for the first time (Jackel, 2002). The approach did not have a name until Nicholas Metropolis, and Stanislav Ulam scientists used it to conduct a multi-dimensional statistical calculation analysis in 1949 and called it the Monte Carlo method (Jackel, 2002). Monte Carlo analysis is based on a computer algorithm to generate sufficiently random numbers to support mathematical 
prediction equation approaching a stochastic process (Jackel, 2002). The following parts are including (1) cost risks minimum-maximum limits, (2) Monte-Carlo simulation approach, (3) Data Mean Shifting Mathematics, and (4) cost risks Monte-Carlo modelling.

\section{LITERATURE REVIEW}

This part of the research will review relevant literatures to justify scientifically the reasons of approaching Monte-Carlo simulation to measure cost risks from clients' perspective. The following literatures is including: (1) Cost Risks Minimum-Maximum Limits and (2) Monte-Carlo Simulation Approach.

\subsection{Cost Risks Minimum-Maximum Limits}

The cost risk range identification, including minimum and maximum limits, is an essential mathematical process before moving to Monte Carlo simulation. It is done after validating all collected data (i.e., risks, impact, and probability). Each validated cost risk has a mean and standard deviation value. This research is using experts' data to simulate actual costs in the future. It will require a minimum sample size equal to 385 for unknown populations using Equation 1 (Sathian et al., 2010). Alternatively, a sample size equal to 400 from published quantitative sample-size tables (Singh \& Masuku, 2014).

$$
\begin{aligned}
& \boldsymbol{n}_{\mathbf{0}}=\frac{\boldsymbol{z}^{\mathbf{2}} \boldsymbol{p} \boldsymbol{q}}{\boldsymbol{e}^{\mathbf{2}}} \\
& \boldsymbol{n}_{\mathbf{0}}=\text { Sample Size } \\
& \boldsymbol{Z}=\text { Standard score } \\
& \boldsymbol{p}=\text { the (estimated) proportion of the population (variability) } \\
& \boldsymbol{q}=1-\text { p } \\
& \boldsymbol{e}=\text { Desired precision level (i.e., } 0.05 \text { for } 95 \% \text { confidence level) }
\end{aligned}
$$

Therefore, the following quantitative minimum required sample calculation of unknown population, using Equation 1, is including $Z=1.96$ (Sathian et al., 2010). It is assuming the population error of confidence level $95 \%$ as e (i.e., $1-0.95=0.05$ ) and it is assuming maximum variability as justified earlier (i.e., $\mathrm{P}=0.5$ ).

$$
\text { Sample Size }=\frac{1.96^{2} * 0.5 * 0.5}{0.05^{2}}=384.16 \approx 385
$$

Alternatively, it is possible to use the Monte Carlo simulation to cover the smaller sample size gaps. This research study is justified in how the acceptable sample size is equal to 55 face-to-face survey interviews. Therefore, this research study does not follow the published table or sample size mathematical calculations, as mentioned in Equation 1. However, it still requires to include accurate mathematical modelling to deliver an accurate final cost estimation model. Monte Carlo simulation can guaranty the high precision of each modelled variable (Heijungs, 2020). It requires upper and lower limits to ensure 
precise accuracy (Heijungs, 2020). These limits need to be around the actual population's mean using standard deviation (Heijungs, 2020). Therefore, adequate sample size and data collection methods ensure accurate means and standard deviations as justified earlier. These data will successfully represent the population's mean and standard deviation, as proved earlier. Therefore, in this research study, Monte Carlo variables' modelling limits are extracted from the validated experts' face-to-face survey interviews to ensure accuracy. Figure 1 clarifies the difference between accuracy and precision (Heijungs, 2020). Valid data will ensure accuracy, and Monte Carlo simulation will ensure the precision of each variable.

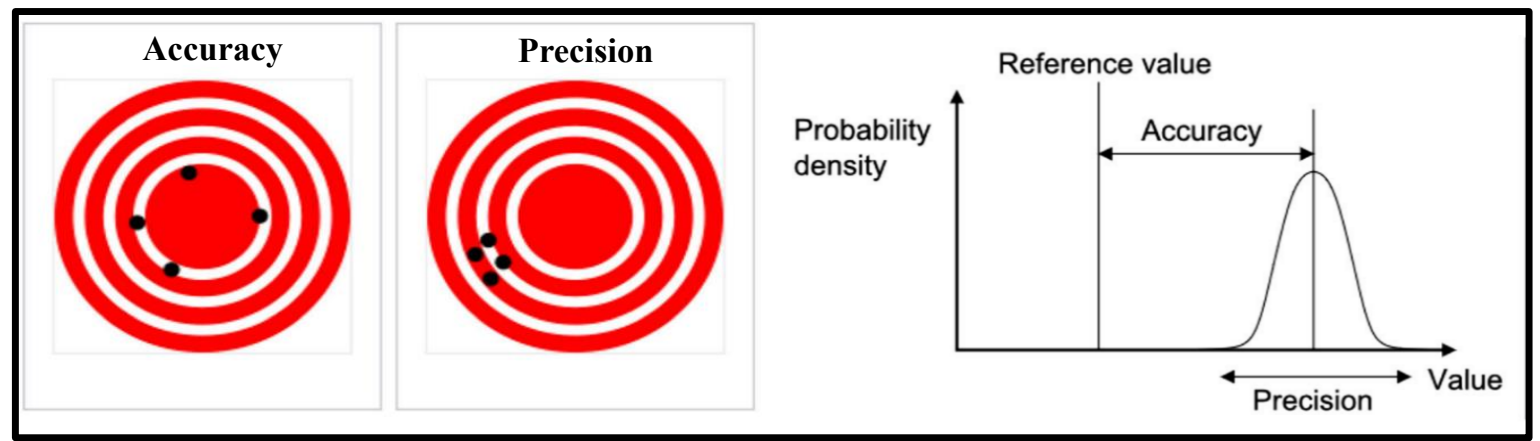

Figure 1. The difference between accuracy and precision (Heijungs, 2020).

Finally, Equation 2 is used to define the Monte Carlo upper and lower limits. The following part will detail how Monte Carlo simulation is accomplished in this research study.

Monte Carlo Upper and Lower Limits = Cost Risk Mean \pm Standard Deviation

\subsection{Monte-Carlo Simulation Approach}

This part will explain how Monte Carlo modelling is validated in this research study. Monte Carlo modelling is the stochastic mathematical estimation modelling of this research study (Bukaçi et al., 2016). This method is used to maximize the likelihood of functions representing actual results at absent data incidence (Caffo et al., 2005). It is also used in previous research to maximize linear equations' likelihood to represent actual data (Lai \& Lin, 2011). Also, researchers proved that it is valuable for conducting unbiased simulations (Thompson \& McLeod, 2009). However, it is found in previous research that Monte Carlo simulation requires a minimum number of iterations (i.e., random values) to validate functions' likelihood of actual representation (Heijungs, 2020; Bukaçi et al., 2016; Caffo et al., 2005; Nowak et al., 2016; Thompson \& McLeod, 2009). It is found that previous research used a wide range of valid Monte Carlo iterations, including 100, 1,000, 10,000, 1x105, and 1x107 (Heijungs, 2020; Nowak et al., 2016; Thompson \& McLeod, 2009). It is also extracted from the literature that Monte Carlo required the number of iterations based on the research type (Heijungs, 2020; Bukaçi et al., 2016; Caffo et al., 2005; Nowak et al., 2016; Thompson \& McLeod, 2009). It is unnecessary to have more accurate outputs by adding more iterations; in other words, previous research proved that using 1 x 105 iterations is more accurate than using $1 \times 107$ iterations for the same study (Thompson \& McLeod, 2009). It is essential to identify the minimum required iteration number in this research. Moreover, in literature, similar life cycle assessment studies declared that it is common to use $\geq 10,000$ iterations for Monte Carlo modelling and recommended this minimum valid number (i.e., 10,000) for life cycle assessment studies (Heijungs, 2020). Therefore, this research study uses 10,000 iterations for each cost risk Monte Carlo simulation without increasing iterations to prove its validity. 
The mathematical modelling equations of this research Monte Carlo simulation require several assumptions to validate it from a client perspective at the pre-construction stage. These assumptions are listed based on what is explained earlier as the following:

- Constructions and projects industry experts provide all survey risks.

- All weighted risks have an appropriate risk management system set by contractors with values equal to what is provided by industry experts. These risk values are identified and assessed successfully by contractors throughout the projects' whole life cycle.

- All risks identified by industry experts have been considered by contractors and included in the contract value cost. Therefore, the contract bill of quantity pricing is done by contractors and is including all identified risks by industry experts.

- The Monte-Carlo simulation will be used to calculate the error in the project's risk management system and find the adjustment value for each risk factor. It is required because the project's actual costs are not matching pre-construction estimated costs as justified earlier. Therefore, this is evidence of having errors in experts identified risk values. Moreover, it will be corrected through this research Monte Carlo method.

- The adjustment values are errors of mean risks, minimum risks, and maximum Risks. It will be used in the final system dynamics model to ensure that the estimated cost includes all its possible impacting risks. This will be including the known risks' weightage identified by experts (i.e., known-known and known-unknown risks). Moreover, it will include the unknown risk error (i.e., unknown-unknown risks) by conducting Monte-Carlo Simulation for each cost risk variable.

- Based on the justified reasons behind considering the error of experts' judgment when estimating costs at the pre-construction stage from a client perspective, the final model's minimum, mean, and maximum risks are the error of minimum, mean, and maximum risks.

The adjusted mean is the sample mean after implementing Monte Carlo outputs. This research assumes that the population mean equal to the adjusted mean after finding out each risk variable's error and embracing it in the variable's sample mean. This declaration is to recommend further research periodically to measure risks error and update it continuously. However, as of this research time, all risks are considered accurate and reflect the population's actual data.

From Figure 1, it is clear why using Monte Carlo simulation is essential. However, shifting data means should be done based on moving the full distribution to avoid discrediting experts' data. The mean only shall be moved toward the believed actual population means $\mu$ without changing each risk's standard deviation. Figure 2 clarifies how this research intends to conduct the required shift successfully.

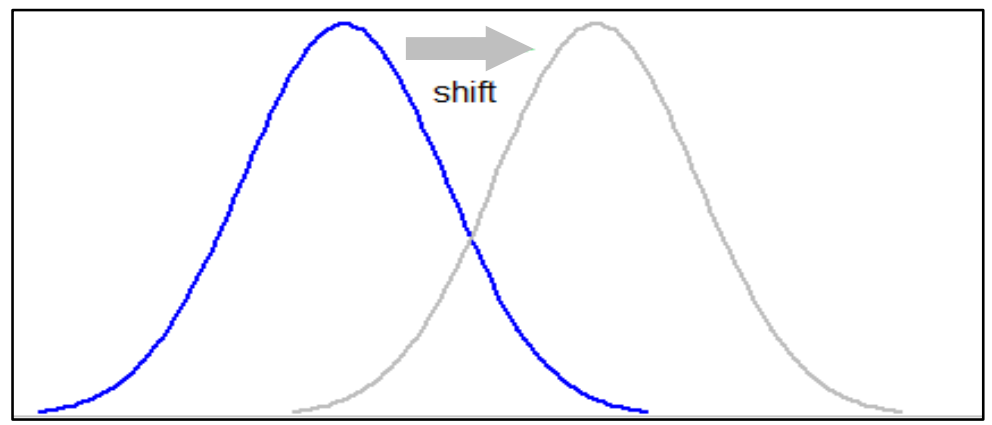

Figure 2. Shifting data mean without discredit distribution reliability should be without changing the standard deviation. 


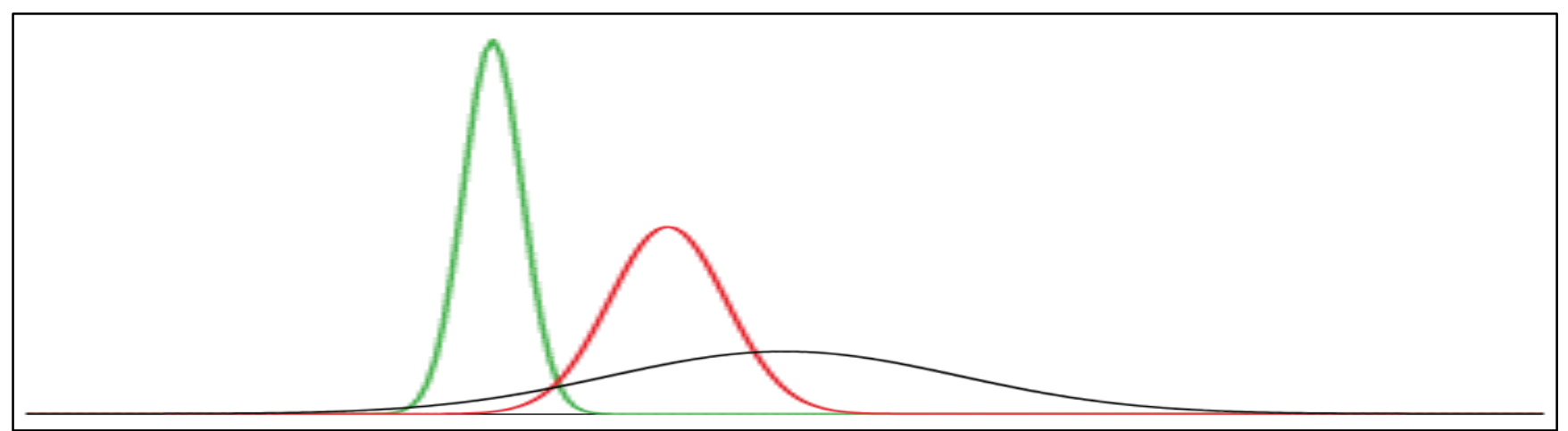

Figure 3. Showing how shifting data mean while changing its standard deviation discredit the full data distribution and withdraw its reliability and validity.

The reason behind fixing each variable's standard deviation is to maintain experts' data reliability and validity. Figure 3 shows how standard deviation change impacts data distribution significantly and withdraws data credibility. However, the normal back distribution has the largest standard deviation. The red-normal distribution has a smaller standard deviation than the black one, but it is larger than the normal green distribution standard deviation. Finally, the green-normal distribution has the smallest standard deviation.

\section{RESEARCH METHODOLOGY}

The research methodology of this article is to develop statistical and mathematical systems using Microsoft Office Excel software Mersenne twister algorithm to satisfy the requirements of the desired Monte-Carlo stochastic approach. Therefore, the research approach in this paper is pure quantitative. This paper approached 55 construction/project experts, in a face-to-face survey interview, to collect quantitively the cost risks values and validated them statistically prior utilizing it in the developed MonteCarlo model.

\section{ANALYSIS AND DISCUSSION}

Monte Carlo cost risks model is developed in this part of the research and analysed using the validated data and the developed equations system. Each raw in the Excel sheet shown in the appendix is a separate Monte Carlo model for the identifies cost risk. The following parts in this section are detailing the analysis processes through a critiqued discussion; however, this is including: (1) Data Mean Shifting Mathematics and (2) Cost Risks Monte-Carlo Modelling.

\subsection{Data Mean Shifting Mathematics}

In this part of the paper, a detailed process of how to satisfy the previously discussed approach mathematically to achieve a successful Monte Carlo Modelling of costs risks. Therefore, the adjusted mean value can be calculated for each risk variable using Equations 3. Then, the adjustment mean value (i.e., error) can be calculated for each risk variable using Equation 4. This process is clarified in Figure 4 for better understanding. 


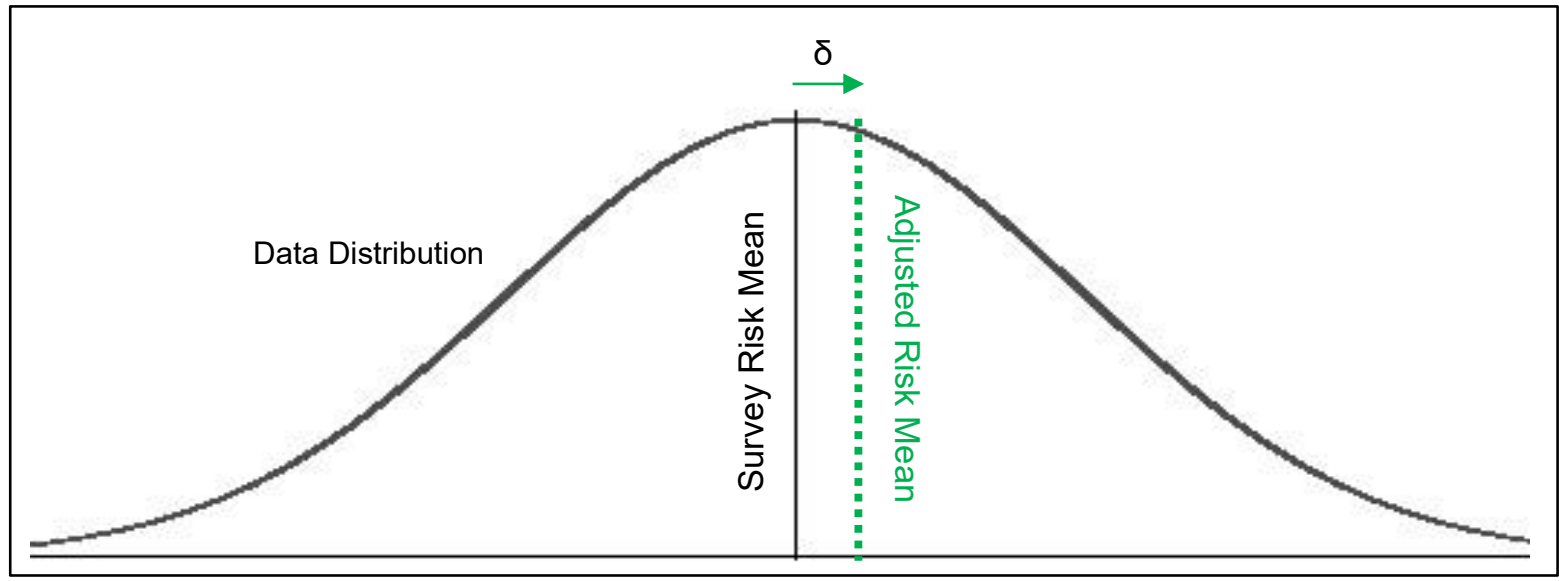

Figure 4. clarify the difference between survey risk mean and adjusted risk mean for the same data distribution of each variable.

Adjusted Mean Value $=\operatorname{Min}+\left[\mathrm{MC}^{*}(\operatorname{Max}-\operatorname{Min})\right]$

Min is the Minimum Survey Risk Value

Max is the Maximum Survey Risk Value

MC is Monte Carlo average of 10,000 random values (between 0 to 1).

Adjusted Mean Value $=\boldsymbol{\delta}=\left|\frac{\boldsymbol{v}_{\boldsymbol{A}}-\boldsymbol{v}_{\boldsymbol{E}}}{\boldsymbol{v}_{\boldsymbol{E}}}\right| * 100$

$\boldsymbol{\delta}$ is the percent error.

$\boldsymbol{v}_{\boldsymbol{E}}$ is the Maximum Survey Risk Value

$\boldsymbol{v}_{\boldsymbol{A}}$ is the Believed actual value (i.e., adjusted value).

After implementing the Monte Carlo simulation method, final system dynamics data inputs can achieve the required accuracy and precision, as shown in Figure 5. This will improve the final cost estimation using the system dynamics approach.

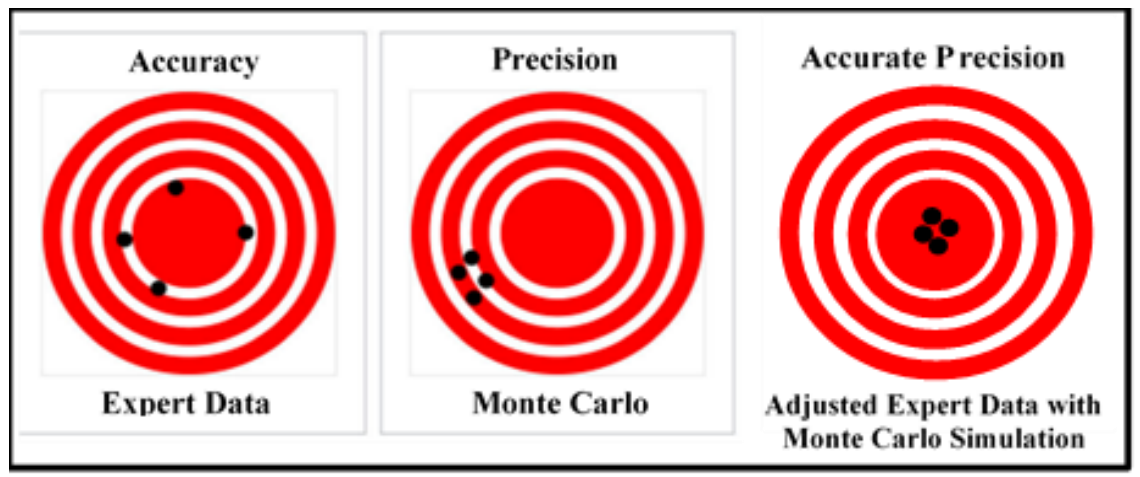

Figure 5. Experts'data and Monte Carlo modelling impact on final outputs' accuracy and precision. 
However, it is found that Adjusted Mean Risk, Adjusted Maximum Risk, and Adjusted Minimum Risk have the same mathematical relationship between Survey Mean Risk, Survey Maximum Risk, and Survey Minimum Risk. This is justified and proved in Equations 5 to 14. These equations clarify how the adjusted minimum and adjusted maximum values are mathematically calculated.

Based on 95\% Confidence:

$\operatorname{Max} \operatorname{Risk}=\overline{\mathrm{X}}+2 \sigma$

Min Risk $=\overline{\mathrm{X}}-2 \sigma$

$\boldsymbol{\sigma}$ is the risk standard deviation

$\overline{\mathbf{X}}$ is the survey sample mean risk

Max Risk is the Normal Distribution Upper Limit

Min Risk is the Normal Distribution Lower Limit

From equations 5 and 6:

$2 \sigma=\operatorname{Max} \operatorname{Risk}-\bar{x}=\bar{X}-$ Min Risk

$2 \bar{x}=$ Min Risk + Max Risk

From equation 4:

Adjustment Mean Value (error) $=\frac{\text { Adjusted Mean }-\overline{\mathrm{x}}}{\overline{\mathrm{x}}}$

By feeding equation 9 into equation 8 and by using mathematical multiplication and division properties:

$2 \overline{\mathrm{x}} * \frac{\text { Adjusted Mean }-\overline{\mathrm{x}}}{\overline{\mathrm{x}}}=($ Min Risk + Max Risk $) * \frac{\text { Adjusted Mean }-\overline{\mathrm{x}}}{\overline{\mathrm{x}}}$

$2 \overline{\mathrm{x}} *$ Adjustment Mean $=($ Min Risk + Max Risk $) *$ Adjustment Mean

2 Adjustment Mean $=\left(\frac{\text { Min Risk }}{\overline{\mathrm{x}}}+\frac{\text { Max Risk }}{\overline{\mathrm{x}}}\right) *$ Adjustment Mean

Adjustment minimum risk value $=\frac{\text { Min Risk }}{\overline{\mathrm{x}}} *$ Adjustment Mean

Adjustment maximum risk value $=\frac{\text { Max Risk }}{\overline{\mathrm{x}}} *$ Adjustment Mean

Therefore, it is mandatory to approach the Monte Carlo method in this research to obtain the minimum, mean, and maximum risks error. This process will have the required 10,000 random iterations generated using the Microsoft Office Excel software program. Then, start computing using equations 5 to 14. 
In order to ensure the implementation of accurate and successful Monte Carlo simulation for the verified data, it is mandatory to understand the basic software mathematics used to generate random numbers. Furthermore, to confirm that chapter 4 developed mathematical modelling equations that can be computed utilizing Excel software. Therefore, by reviewing the Microsoft Office Excel official manual, it has been found that Excel RAND() function is the approach to generate random numbers between 0 and 1 (RAND function, 2020). It is also stated clearly that RAND() function uses the Mersenne twister algorithm to generate random numbers (RAND function 2020). According to the literature, the Mersenne twister algorithm is one of many computer algorithms to generate pseudorandom outputs (Self \& Mackey, 2016; Graham \& Talay, 2013). According to the linear feedback shift register, the Mersenne twister is one of the most important random number generating algorithm based on its historical strength (Self \& Mackey, 2016). It has been proved accurate for linear congruential generation and linear functions (Self \& Mackey, 2016). Therefore, this research will use the Microsoft Office Excel software program to approach Monte Carlo stochastic mathematical modelling to compute risks error using Figure 6 algorithm and equations 15 and 16.

$$
\begin{aligned}
& \text { Adjusted Mean Using Excel }=\operatorname{RAND}()^{*}(\mathrm{~b}-\mathrm{a})+\mathrm{a} \\
& =\frac{\sum_{1}^{10,000} R A N D()}{10,000} *(b-a)+a \\
& \mathrm{a}=\text { the Minimum Survey Risk Value } \\
& \mathrm{b}=\text { the Maximum Survey Risk Value }
\end{aligned}
$$

$\operatorname{RAND}()=$ Monte Carlo pseudo-random values (between 0 to 1$)$.

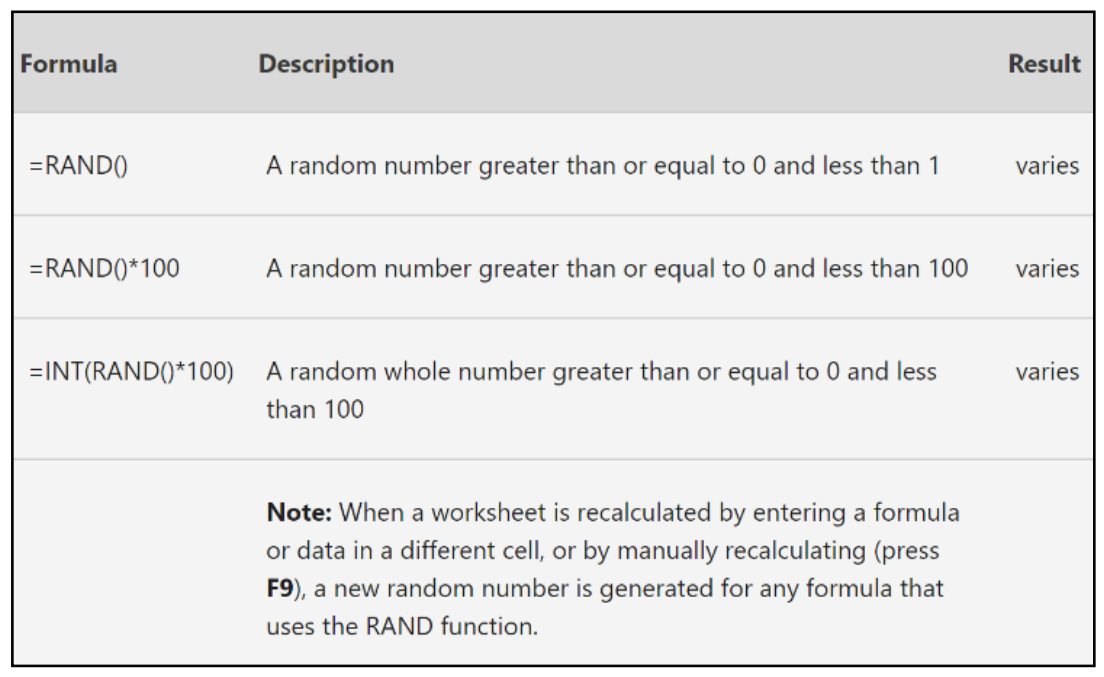

Figure 6. Microsoft office official random equation used in excel (RAND function. 2020).

This research data's calculated error is based on normally distributed data as explained and justified earlier. It will be taken based on using distribution properties (i.e., Mean and Standard Deviation) to apply risks' error impact on the contract value at the design stage to estimate final costs and cashflows. Therefore, each error set related to a variable requires to have the same behavior of its data (i.e., normal distribution 
mean, standard deviation, upper limit, and lower limit). The detailed process shown in Figure 7 must be followed and reflected in the analysis to satisfy this requirement. Otherwise, the mean risk might be lower than the upper limit or more than the upper limit. The procedure shown in Figure 7 ensures that mathematical statistics theory applies on a higher generalization level. Similar research about buildings' operations and management used this approach to check if a theory's relevance can be extended (Meredith, 1998). Researchers' evidence, for approaching testing theories' relevance extension, is based on replacing Newton's gravity theory with Einstein's relativity theory due to generalization (Meredith 1998). Previous literature declared that weighted cost-risks measurement in a project's delivery is achieved by conducting a sensitivity analysis to measure its relativity using a risk assessment probabilistic approach (Akinyemi et al., 2009). It is essential to understand the driving risk force to assign it as the relativity gravity center of each problem (Flores-Colen et al., 2010). Gravity term is used because objects' behavior relatively changes toward each other concerning their response to gravity force. Relativity change in this research can be defined as the change of data errors (i.e., $\Delta$ Minimum and $\Delta$ Maximum) using $\Delta$ Mean as the main driving force connecting all data distribution changes relative to its change.

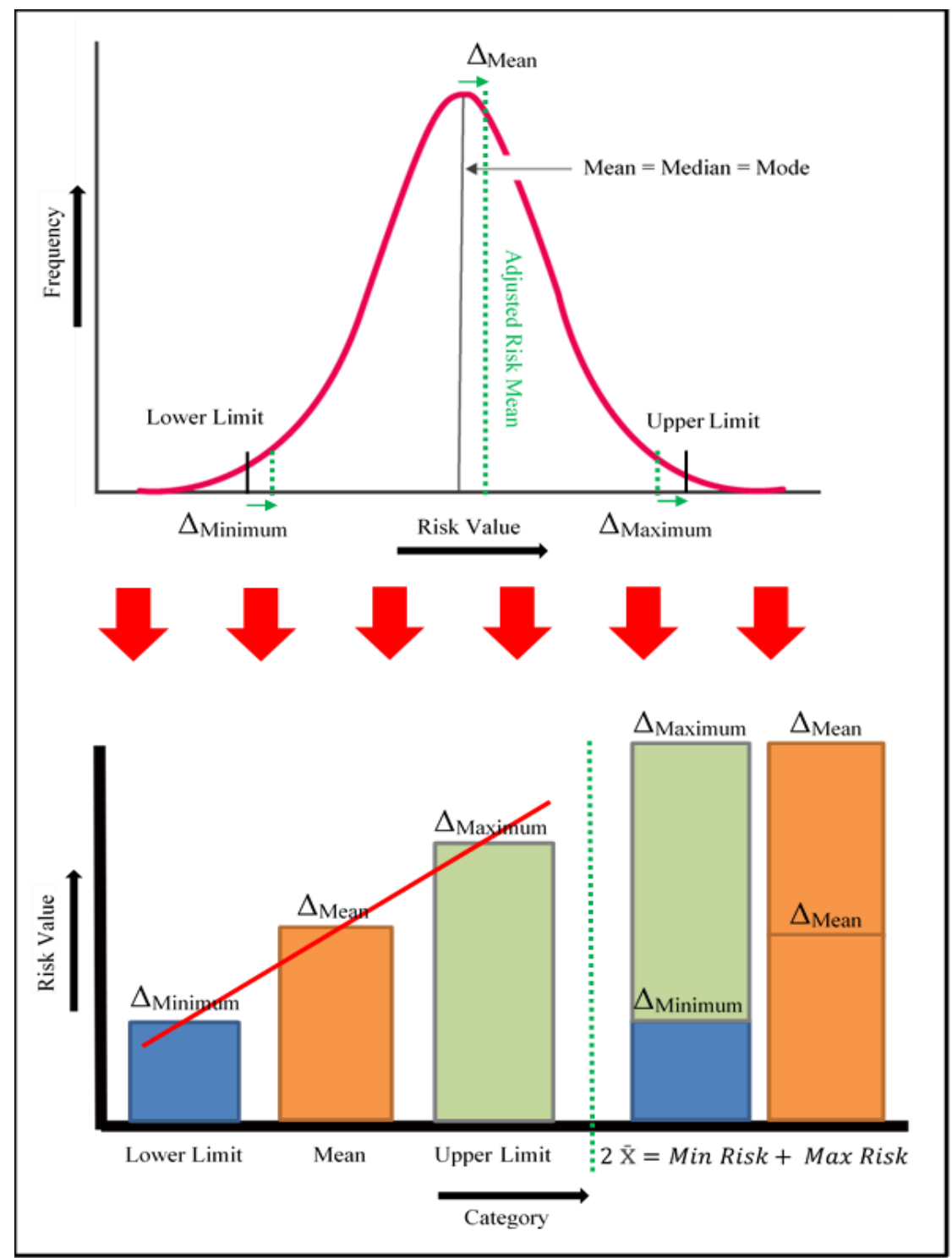

Figure 7. Computing $\Delta$ Minimum and $\Delta$ Maximum using $\Delta$ Mean and equations 9 to14. 


\subsection{Cost Risks Monte-Carlo Modelling}

According to researchers, to identify the accuracy of parameters and attributes measurements, it is most recommended to have a stepwise regression followed by a Monte Carlo simulation (Yang et al., 2019). Built environment projects have cost estimation issues due to client requirements (Ahiaga-Dagbui \& Juffermans, 2019; Oswald et al., 2020). This requires having an estimation model from client perspectives. Monte-Carlo calculation of adjusted and adjusting risks is based on the final SPSS validated data outputs.

In the appendix table, Monte Carlo modelling is fully detailed to obtain the final desired outputs (i.e., risks errors). The first column from the left side is including the serial numbers of each cost risk and the alphabet letter of each variables' group, as shown in Figure 8. However, in Figure 8, the risk description is represented in the second column of the appendix table, and its reference is CA1 (i.e., C = CAPEX, A $=$ Group Symbol, and $1=$ the activity number under the group).

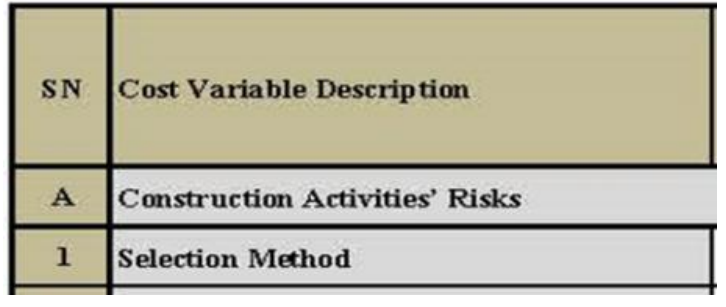

Figure 8. Cost risks description and reference code (i.e., CA1) from the appendix table.

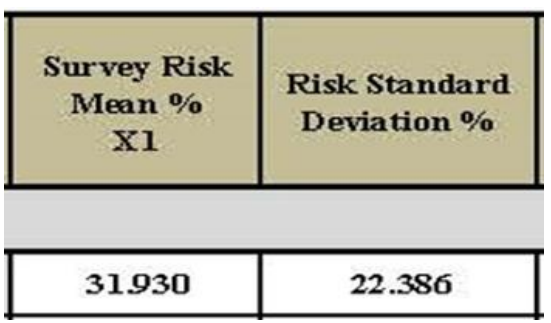

Figure 9. CA1 Cost risks SPSS Mean and Standard Deviation from the appendix table.

The third and fourth columns are the survey risk mean and standard deviation after validating data using SPSS software, as shown in Figure 9. However, to facilitate clarifying further coming calculations in the appendix Table, the survey risk mean column is given a symbol X1 as shown in Figure 9. After that, the lower and upper limits are calculated, as shown in Figure 10, using equations 5 and 6 fifth and sixth column of the appendix table. However, they are given symbols Xmin and Xmax, respectively, to facilitate further calculations understanding of Monte Carlo simulation. In Figure 11, the seventh and eighth columns present the average percentage of 10,000 random values used in Monte Carlo simulation and the adjusted Mean value (i.e., X2 in the appendix table) using equation 9.

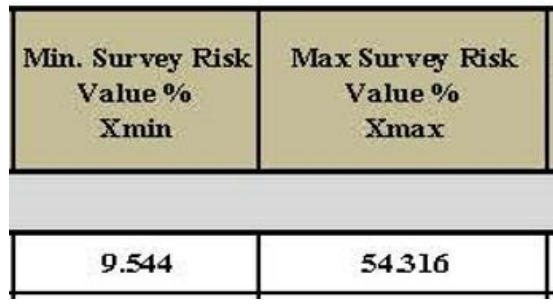

Figure 10. CA1 Cost risks Lower and Upper Limits from the appendix table.

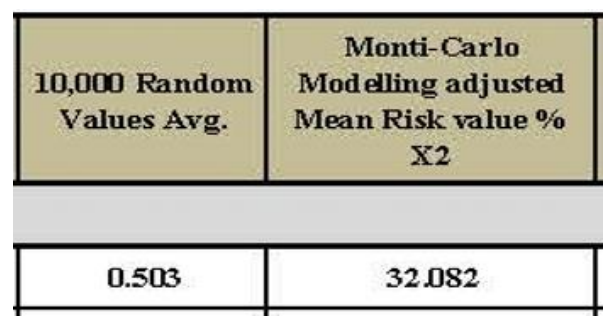

Figure 11. CA1 10,000 iterations average \& cost risks adjusted Mean from the appendix table.

\begin{tabular}{|c|c|c|}
\hline $\begin{array}{l}\text { Relativity Change Between Survey } \\
\text { Minimum Risk Value \% and Survey } \\
\text { Mean Risk value \% } \\
\text { Rmin }=(\text { Xmax } / \mathrm{X} 1)^{\star} \text { Rmean }\end{array}$ & $\begin{array}{l}\text { Relativity Change Between Survey } \\
\text { Maximum Risk Value \% and } \\
\text { Survey Mean Risk value \% } \\
\text { Rmax }=(\mathrm{Xmax} / \mathrm{X} 1)^{\star} \text { Rmean }\end{array}$ & $\begin{array}{l}\text { Relativity Change Between Monti-Carlo } \\
\text { Modelling adjusted Risk Mean Value \% } \\
\text { and Survey Mean Risk value \% } \\
\text { Rmean=ABS }(\mathrm{X} 2-\mathrm{X} 1) / \mathrm{X} 1\end{array}$ \\
\hline 0.001422906 & 0.008097921 & 0.004760413 \\
\hline
\end{tabular}

Figure 12. CA1 $\triangle$ Minimum, $\triangle$ Mean, and $\triangle$ Maximum from the appendix table. Calculations are using equations 9 to 14 and figure 7 . 
Then, the calculations of mean error and the relativity change of data upper and lower limits, as explained earlier, is done using equations 9 to 12, as shown in Figure 12. However, these calculations occur in each cell of the ninth, tenth, and eleventh columns of the appendix table, respectively. However, the equations used in each of these columns use introduced symbols mentioned previously in the appendix table column for better understanding, as shown in Figure 12.

Finally, in Figure 13, the difference between validated data survey means and adjusted Monte Carlo simulation means is presented graphically and numerically, respectively, in the twelfth column. It is used to have a balanced random value's average by ensuring almost $50 \%$ of the differences under positive and negative changes. This will avoid any bias with or against experts provided data, as shown in Figure 14.

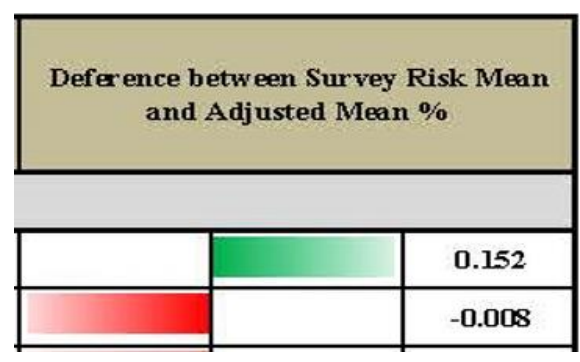

Figure 13. CA1 and CA2 Cost risks deference between the survey and the adjusted Means from the appendix table.

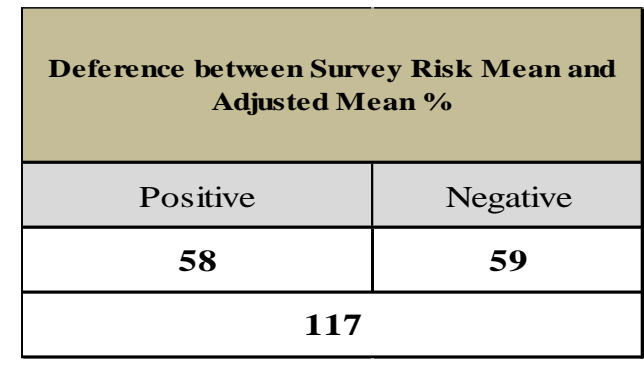

Figure 14. Balancing the appendix table Cost risks deference between the survey and the adjusted Means.

The model is finalized after simulating several random iterations and fixed the values that caused 58 positives and 59 negatives (i.e., almost $50 \%$ in positive and $50 \%$ in negative) as counted in the last column of the appendix table and as shown in Figure 14. After that, from Figures 8 to 13, the appendix table is explained using the same cost risk (i.e., under CAPEX, construction activities group, selection method risk $=\mathrm{CA} 1=$ first raw). Thus, the Monte Carlo simulation of this risk will be detailed as an example of what the appendix table data reflects. The most important part is how columns nine to eleven calculate relativity change using Figure 7 approach. From using equation 8 (i.e., $2 \overline{\mathrm{x}}=$ Min Risk + Max Risk) Figure 12 shows that $\mathrm{R}_{\min }$ (i.e., risk lower limit relativity change) is equal to 0.001422906 . Furthermore, $\mathrm{R}_{\max }$ (i.e., risk upper limit relativity change) is equal to 0.008097921 . Moreover, $\mathrm{R}_{\text {mean }}$ (i.e., risk's adjustment Mean) is equal to 0.004760413 . Therefore, equation 8 can be satisfied through the following substitution.

$$
\begin{gathered}
2 * 0.004760413=0.001422906+0.008097921 \\
\sqrt{0.00952083=0.00952083}
\end{gathered}
$$

Each row in the appendix table is a Monte Carlo Simulation for each risk, as explained in this article.

\section{CONCLUSION}

In summary, this article introduced the history of Monte Carlo modelling and its importance in estimating unknown numerical values using a probabilistic stochastic approach. Also, it is clarified how the Monte Carlo simulation relates to cost-risks outputs. Therefore, this article is presenting 117 Monte Carlo simulations to end with the 117 values of Rmin, Rmean, and Rmax to be used in system dynamics cost modelling. 


\subsection{Originality}

According to the best knowledge of the author, there is no previous research approached identifying residential projects whole life cycle cost-risks, from client perspective at the preconstruction stage, using Monte-Carlo simulation approach.

\subsection{Theoretical Implications}

This article output is considering the cost-risks from client perspective after obtaining the tender contract value from contractors. The project's contract value is including identified cost risks and its assessment costs by contractor's experts. Further research is recommended to investigate the reason of project cost estimation error compared with the actual completion costs.

\subsection{Practical Implications}

This article provides Monte-Carlo approach to measure the error to adjust estimated contractual costs and predict the actual cost at the end of the project. Project management professional.

\subsection{Limitations and Future Research}

The limitations of this research have been collected and summarized as the following:

1- The collected data was based on experts' face-to-face survey interviews and Monte Carlo stochastic modelling. It is recommended to investigate the model response after using published tables sample size for data collection. Experts identified risk values against previous historical completed projects (i.e., at least 30 projects) to calculate each risk error and adjust risk value feed into the system dynamics cost model.

2- Finally, this research included, in Monte Carlo simulation, 10,000 iterations as the minimum required iterations. However, according to the researcher's best knowledge, no one used system dynamics for cost modelling in previous research. It is recommended to investigate the impact of changing the Monte Carlo iterations number on the developed system dynamics cost modelling accuracy.

\section{REFERENCES}

[1] Ahiaga-Dagbui, D. and Juffermans, T. (2019). DEVELOPING COLLABORATIVE AND LESS ADVERSARIAL PROJECT TEAMS, THE BUILDING ECONOMIST, vol. 3(March), pp. 43-47.

[2] Akinyemi, B., Ojiako, U., Maguire, S., Steel, G. and Anyaegbunam, A. (2009). Nigerian Banks and the Perception of Risk in PPP Project Delivery, Journal of Finance and Management, vol. 8(2), pp. 1-20.

[3] Bukaçi, E., Korini, T., Periku, E., Allkja, S. and Sheperi, P. (2016). Number of iterations needed in Monte Carlo Simulation using reliability analysis for tunnel supports, Int. Journal of Engineering Research and Applications, vol. 6(6), pp. 60-64.

[4] Caffo, B. S., Jank, W. and Jones, G. L. (2005). Ascent-based Monte Carlo expectation - maximization, Royal Statistical Society, vol. 67(2), pp. 235-251.

[5] Flores-Colen, I., de Brito, J. and Freitas, V. (2010). Discussion of Criteria for Prioritization of Predictive Maintenance of Building Façades: Survey of 30 Experts, Journal of Performance of Constructed Facilities, vol. 24(4), pp. 337-344.

[6] Graham, C. and Talay, D. (2013). Stochastic Simulation and Monte Carlo Method, Springer Heidelberg.

[7] Heijungs, R. (2020). On the number of Monte Carlo runs in comparative probabilistic LCA, The International Journal of Life Cycle 
Assessment, vol. 25(1), pp. 394-402.

[8] Jackel, P. (2002) Monte-Carlo methods in finance, Interscience.wiley.com. [online]. [Accessed 10 October 2020]. Available at: http://www3.interscience.wiley.com/journal/114179393/abstract.

[9] Lai, G. and Lin, X. (2011). Condition for relaxed Monte Carlo method of solving systems of linear equations, Supercomput, vol. 57(1), pp. 256-264.

[10] Meredith, J. (1998). Building operations management theory through case and field research, Journal of Operations Management, vol. 16(4), pp. 441-454.

[11] Nowak, M., Miao, J., Dumonteil, E., Forget, B., Onillon, A., Smith, K. S. and Zoia, A. (2016). Annals of Nuclear Energy Monte Carlo power iteration: Entropy and spatial correlations, Annals of Nuclear Energy, vol. 94(1), pp. 856-868.

[12] Östlund, U., Kidd, L., Wengström, Y. and Rowa-Dewar, N. (2011). Combining qualitative and quantitative research within mixed method research designs: A methodological review, International Journal of Nursing Studies, vol. 48(3), pp. 369-383. doi

[13] Sathian, B., Sreedharan, J., Baboo, N. S., Sharan, K., Abhilash, E. S. and Rajesh, E. (2010). Relevance of Sample Size Determination in Medical Research, Nepal Journal of Epidemiology, vol. 1(June), pp. 4-10.

[14] Self, J. and Mackey, M. C. (2016). Random Numbers from a Delay Equation, Journal of Nonlinear Science, vol. 26(5), pp. $1311-1327$.

[15] Singh, A. and Masuku, M. (2014). SAMPLING TECHNIQUES \& DETERMINATION OF SAMPLE SIZE IN APPLIED STATISTICS RESEARCH : AN OVERVIEW, International Journal of Economics, Commerce and Management, vol. 2(11), pp. 1-22.

[16] Support.microsoft.com. (2020). RAND Function. [online]. [Accessed 19 January 2020]. Available at: $<$ https://support.microsoft.com/en-us/office/rand-function-4cbfa695-8869-4788-8d90-021 ea9f5be73?ui=en-us\&rs=en-us\&ad=us>

[17] Thompson, K. and Mcleod, A. (2009). Accelerated ensemble Monte Carlo simulation, CUTTING EDGE CREDIT RISK, vol. 4(April), pp. 86-92.

[18] Yang, P. Y., Hui, C. J., Tien, D. J., Snowden, A. W., Derfus, G. E. and Opel, C. F. (2019). Accurate definition of control strategies using cross validated stepwise regression and Monte Carlo simulation, Journal of Biotechnology, vol. 2(November), pp. 1-10. 
Journal of Entrepreneurship and Project Management ISSN 2520-9116 (Online) 
Journal of Entrepreneurship and Project Management

ISSN 2520-9116 (Online)

Vol. 6, Issue No.1, pp 72 -89, 2021

www.carijournals.org

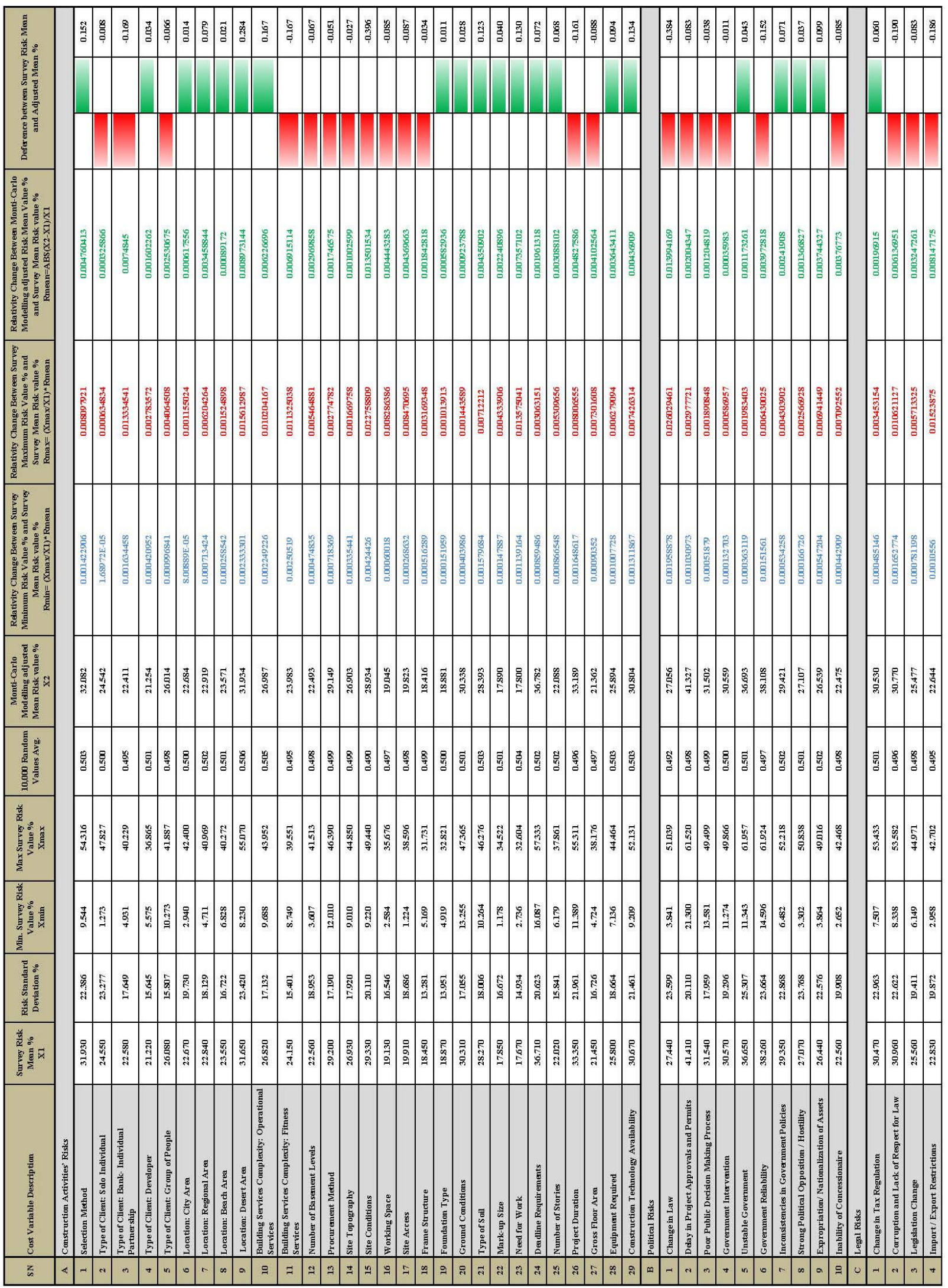


Journal of Entrepreneurship and Project Management

ISSN 2520-9116 (Online)

Vol. 6, Issue No.1, pp 72 -89, 2021

www.carijournals.org

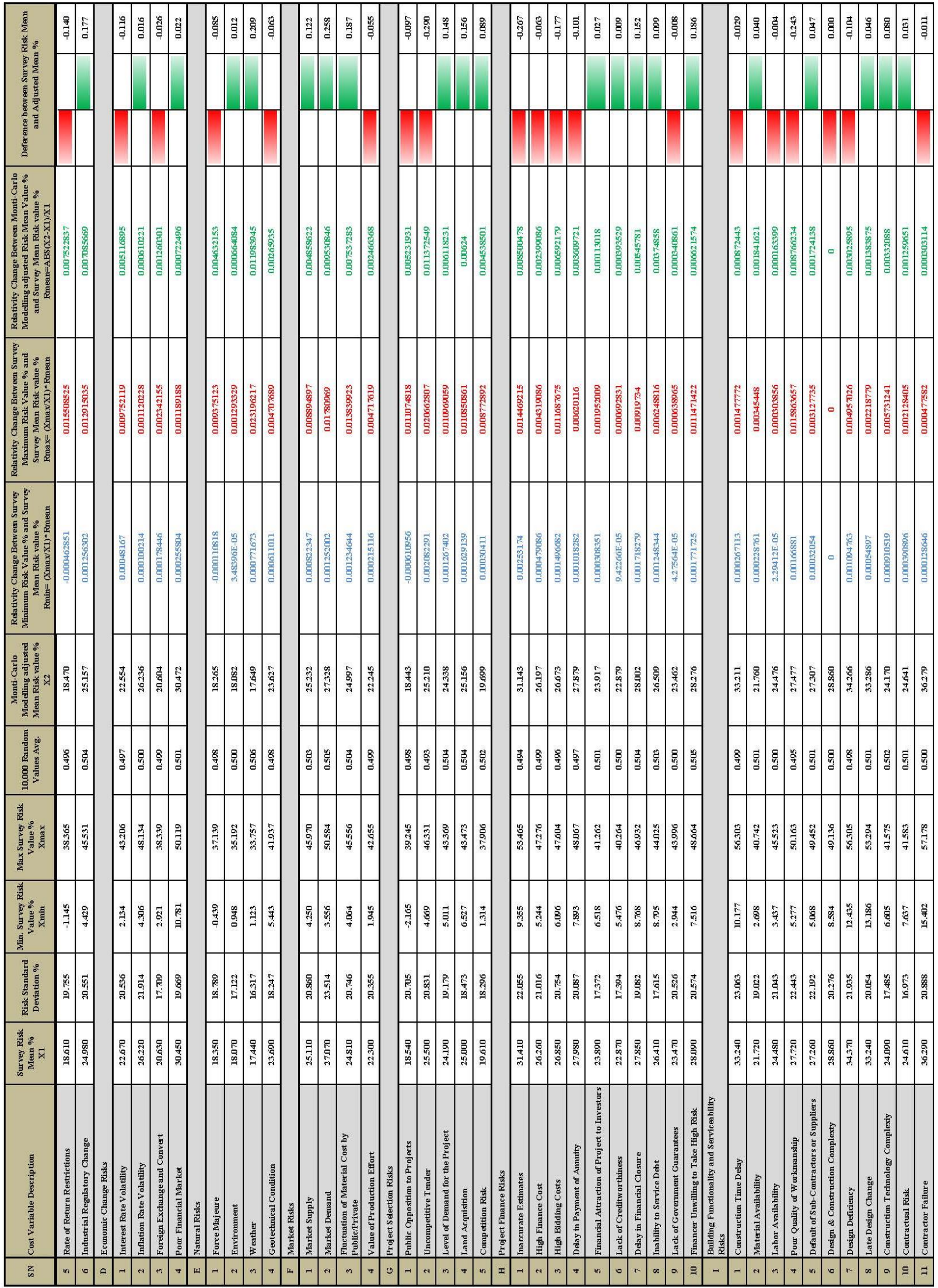


Journal of Entrepreneurship and Project Management

ISSN 2520-9116 (Online)

Vol. 6, Issue No.1, pp 72 -89, 2021

www.carijournals.org

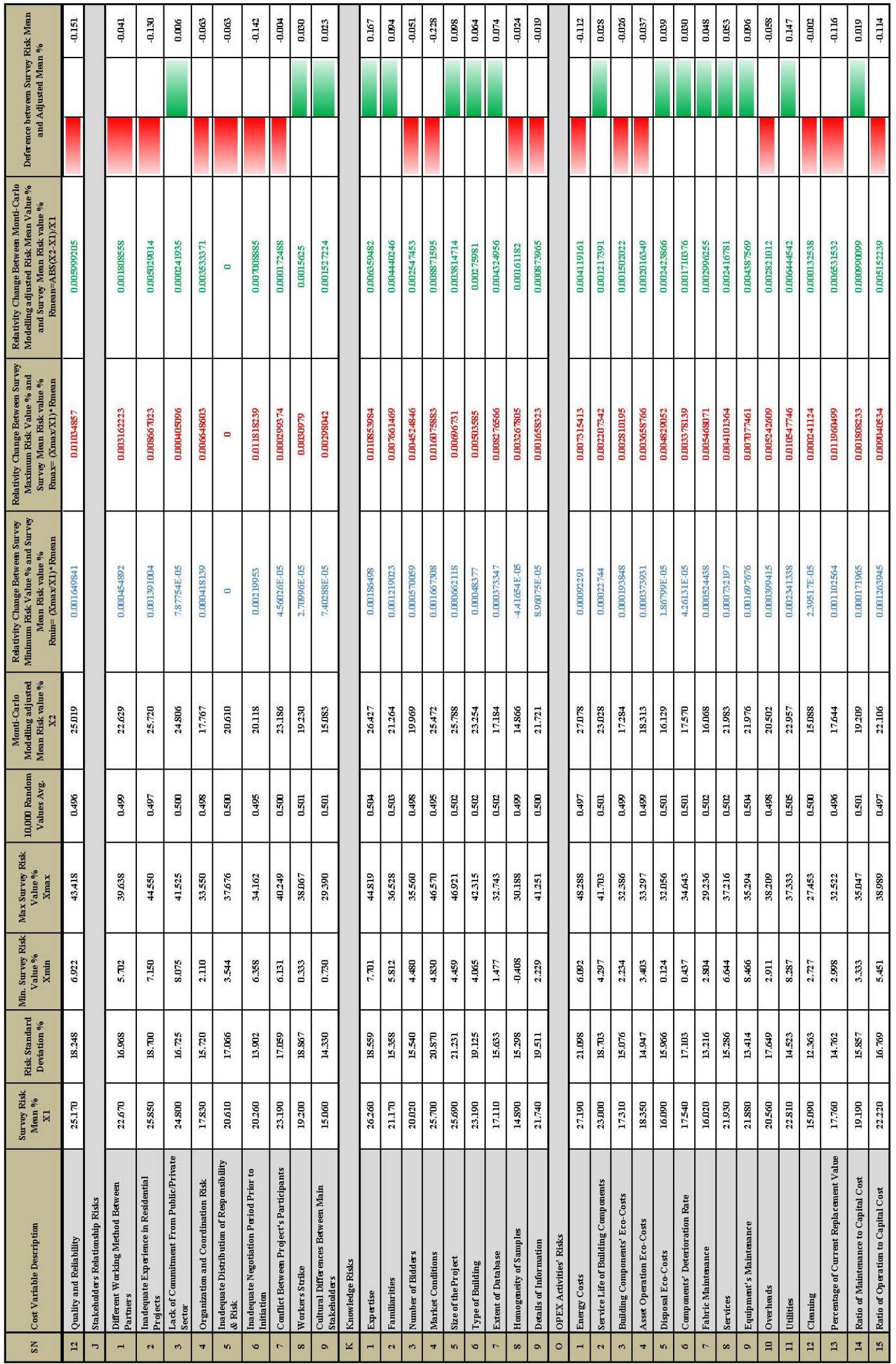

